\title{
Etiology of Keratoconus: proposed biomechanical pathogenesis
}

\author{
Roy Asher ${ }^{1}$, Amit Gefen $^{1 *}$, Elad Moisseiev ${ }^{2}$ and David Varssano ${ }^{2}$
}

\begin{abstract}
Background: The etiology of keratoconus most likely involves substantial biomechanical interactions. The goal of this study was to characterize corneal biomechanics using computer modeling techniques in order to elucidate the pathogenesis of keratoconus in biomechanical terms.

Methods: Finite element models of the cornea that are based on anatomical dimensions were developed. Cases comprising of thinned regions as well as regions with degraded isotropic mechanical properties and a case of gradual stiffening towards the limbus were subjected to normal intraocular pressures. The resulting deformations and dioptric power maps were analyzed and compared. Three additional cases that are based on a model of a thin plate were used to demonstrate the effect a transition from orthotropic to isotropic mechanical properties would have in terms of deformations and diopteric power maps.
\end{abstract}

Results: Results show that under $10 \mathrm{mmHg}$ intraocular pressure, decreasing the modulus of elasticity and thinning have opposite effects on the dioptric power maps of a homogenous isotropic cornea. When the thickness was maintained at 500 microns and the stiffness was decreased from $0.4 \mathrm{MPa}$ to $0.04 \mathrm{MPa}$ there was an increase of more than 40 diopters. For a cornea with a constant modulus of elasticity value of $0.4 \mathrm{MPa}, 350$ microns decrease in thickness resulted in a decrease of approximately 25 diopters. The anisotropic non-homogenous characteristics of the cornea have shown to be critical for maintaining the morphology of a healthy corneal.

Conclusions: Degradation of the circumferential fibers may very well be an initiating factor of a biomechanical process in which a bulge is gradually created from a presumably healthy cornea under normal underlying pressures and therefore, the identification of the early stages of keratoconus might be achievable by monitoring the in-vivo corneal fiber distribution.

Keywords: Etiology; Pathogenesis; Keratoconus; Biomechanics; Finite elements; Diopter

\section{Background}

The cornea forms the transparent outer covering of the visible colored portion of the eyeball. Normal corneas have a horizontal diameter (white to white) in the range of $11-12 \mathrm{~mm}$ in $95 \%$ of the cases [1-3]. The boundary of the cornea is referred to as the limbus region. The central area that lies directly in front of the pupil is the main optical zone and is about 3-4 $\mathrm{mm}$ in diameter. In healthy cases the radius of curvature of the central cornea has been reported to be $7.86 \pm 0.26 \mathrm{~mm}$ (mean \pm standard deviation). The central corneal thickness is

\footnotetext{
* Correspondence: gefen@eng.tau.ac.il

${ }^{1}$ Department of Biomedical Engineering, Faculty of Engineering, Tel Aviv University, Tel Aviv 69978, Israel

Full list of author information is available at the end of the article
}

$0.52 \pm 0.04 \mathrm{~mm}$. The cornea thickens toward its periphery, where its value is about $0.65 \mathrm{~mm}$ [1-3].

The corneal stroma is composed of approximately 300-500 lamellae of collagen fibrils that form most of the corneal tissue and therefore has a major influence on the corneal biomechanics. The directionality of the collagen fibrin in different parts of the cornea has implications on its shape. X-ray scattering studies have indicated that the preferred orientation is more prevalent in the posterior half of the stroma $[4,5]$. In one study, wide-angle $\mathrm{X}$-ray scattering was used in order to quantify the relative number of stromal collagen fibrils directed along two preferred corneal lamellar directions. Approximately onethird of the fibrils throughout the stromal depth tend to lie within a $45^{\circ}$ sector of the superior-inferior meridian,

\section{它}


and similarly for the nasal-temporal direction. The data suggest that, on average, the two directions are populated in equal proportion at the corneal center. An annulus of collagen fibers that encircle the limbus was also observed. It has been proposed that the preferential fibril orientation exists in order to take up the stress of the ocular rectus muscles along the cornea $[4,5]$. The complex enforcements emphasize the importance of knowing how the existence and orientation of collagen fibrils influences the overall corneal mechanical behavior [6].

Injury and disease can alter the thickness, curvature and transparency of the cornea, leading to severe changes in visual functionality. Keratoconus is a disease of the cornea which involves non-inflammatory changes to the corneal microstructure as well as to its macrostructure. As a result, the cornea gradually develops a conic-like shape, with serious implications on its refractive power [7-9]. Most keratoconic corneas have characteristically large differences between their central and peripheral thicknesses compared to normal corneas. Though keratoconus is a not a common condition, it may develop from a presumably normal cornea under normal intraocular pressure (IOP) values [10]. The pathogenesis of this disease is unclear, and several factors have been suggested to be involved in it, including prolonged elevated IOP, bursts of intense force, overexposure to sunlight, improper fitting of contact lenses, eye rubbing, decreased corneal tissue strength, and decreased corneal tissue mass [1,9]. In mild conditions visual acuity (VA) can be corrected using glasses or rigid gas permeable contact lenses but severe cases may require corneal transplantation.

Most corneal topography systems do not plot surface elevations but describe the surface in terms of dioptric values. These dioptric maps are popular because they use the terminology of keratometry which clinicians are familiar with. Just as different elevation maps of the same cornea can be drawn, dioptric data for the same surface can also be expressed. Though these maps are also popular among clinicians and are widely used in optometry to quantify surface curvature and surface refractive power, they are rarely extracted with computational modeling post-inflation although they can provide a deep understanding of the corneal biomechanics [1].

We hypothesize that the etiology of keratoconus could be based solely on substantial biomechanical interactions. It has been previously purposed that pathological changes of the tissue structure might alter the mechanical properties of the cornea, which in turn can affect the gross shape of the cornea under IOP [1]. Therefore, the objective of the current study is to provide a better insight into corneal biomechanics using finite element modeling (FEM) analysis along with corresponding post-deformation dioptric power maps in order to illuminate the etiology of this poorly understood condition.

\section{Methods}

\section{Geometry and mechanical properties}

A three-dimensional nominal model of the cornea, for later on producing model variants as described below, was created using commercial CAD software (Dassault Systèmes, SolidWorks Corp., Solidworks 2012). The geometric properties that were defined are shown in Figure 1 and are based on the available literature $[9,11]$. The anterior surface radius of curvature was set to $7.8 \mathrm{~mm}$. The horizontal diameter and height were set to $11 \mathrm{~mm}$ and $2.4 \mathrm{~mm}$ respectively [12].

The nominal case was altered using the + CAD and ScanIP modules of Simpleware (Simpleware, 2012) resulting in ten distinct cases. These comprise of thinned regions (cases \#2-4 and case \#8) as well as regions with degraded mechanical properties (cases \#5-7 and case \#9) and a case of gradual stiffening towards the limbus (case \#10). Detailed properties and schemes of these cases are presented in Table 1 and Figure 2 respectively [1,9].

A transition from orthotropic (different mechanical properties in three directions) to isotropic (uniform mechanical properites in all orientations) through transversely isotropic (mechanical properties are symmetric about an axis that is normal to a plane of isotropy) mechanical properties would alter the displacements and dioptric power maps. Such transition might be attributed to a change in the corneal fiber disstribution. To study this transition in a preliminary configuration, a model of a thin square plate with a thickness of $0.7 \mathrm{~mm}$ and sides of 6 $\mathrm{mm}$ was created. A $2 \mathrm{~mm}$ diametered weak region was created in the center of the plate as depicted in Figure 3.

Three cases (cases \#11-13) were defined based on the plate model by setting the mechanical properties to isotropic (case \#11), transversaly isotropic (case \#12) and orthotropic (case \#13). The values that were used with the transversely isotropic and orthotropic cases are summarized in Table 2 [1]. Isotropic mechanical properties were defined as in the nominal case (case \#1).

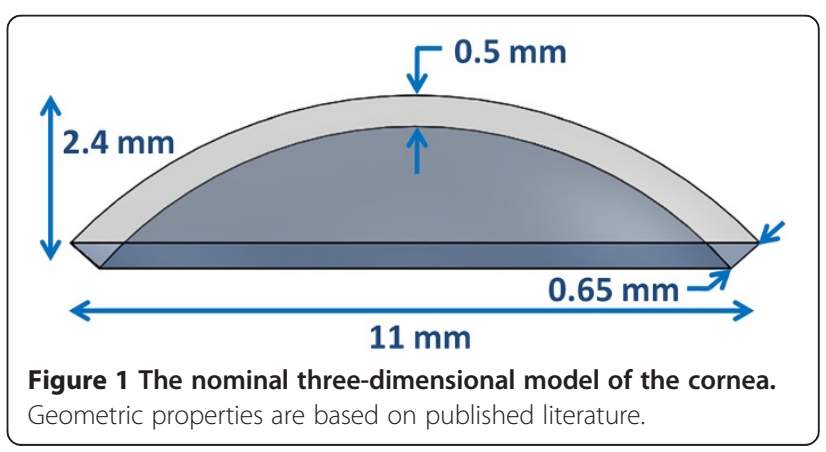


Table 1 A summarization of the geometric and mechanical properties of cases \#1 - 10

\begin{tabular}{|c|c|c|c|c|c|}
\hline & \multirow[t]{2}{*}{ Description } & \multicolumn{2}{|c|}{ Geometry } & \multicolumn{2}{|c|}{ Mechanical properties } \\
\hline & & Minimal thickness $[\mu \mathrm{m}]$ & Region diameter $[\mathrm{mm}]$ & Elastic modulus [MPa] & Region diameter $[\mathrm{mm}]$ \\
\hline Case 1 & Nominal & 500 & - & 0.4 & - \\
\hline Case 2 & Mildly thin & 350 & 2 & 0.4 & - \\
\hline Case 3 & Severely thin & 140 & 2 & 0.4 & - \\
\hline Case 4 & Small defect & 350 & 0.35 & 0.4 & - \\
\hline Case 5 & $50 \%$ more compliant & 500 & - & 0.2 & 4 \\
\hline Case 6 & 70\% more compliant & 500 & - & 0.12 & 4 \\
\hline Case 7 & $90 \%$ more compliant & 500 & - & 0.04 & 4 \\
\hline Case 8 & Off-center thin & 250 & 2 & 0.4 & - \\
\hline Case 9 & Off-center more compliant & 500 & - & 0.04 & 2 \\
\hline \multirow[t]{4}{*}{ Case 10} & Concentric stiffening & 500 & - & 0.35 & 5 \\
\hline & & & & 0.6125 & $5-6$ \\
\hline & & & & 0.875 & $6-7.5$ \\
\hline & & & & 1.225 & 7.5-11 \\
\hline
\end{tabular}

The constitutive relation between stresses and strains for the orthotropic cases is presented in the following equation:

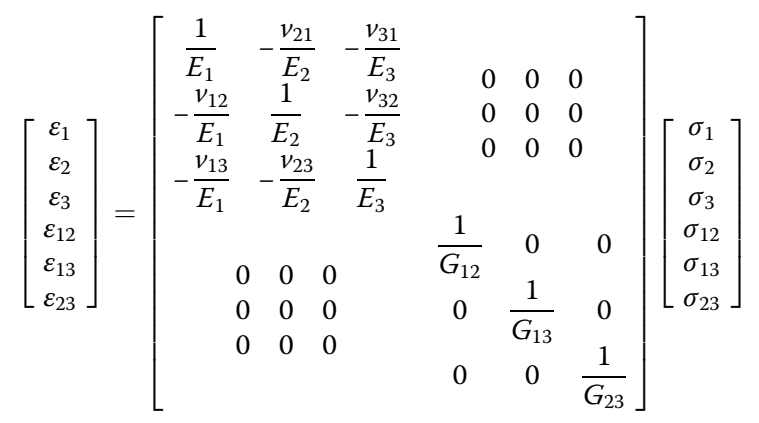
(1) were set as fixed at the perimeter's surface and the $10 \mathrm{mmHg}$. In cases \#11-13 the boundary conditions

Where $E_{i j}$ corresponds to the elaic modulus in each of the three principal material directions, $E_{i j}$ are the three Poisson's ratios that represent the coupling between axial and transverse strains, and $G_{i j}$ are the three shear moduli that represent the relationship between the three modes of shear stress and strain.

Boundary conditions and loading Boundary conditions for cases \#1 -10 were set as fixed along the limbus surface and elements on the posterior surface of the cornea were subjected to an IOP of

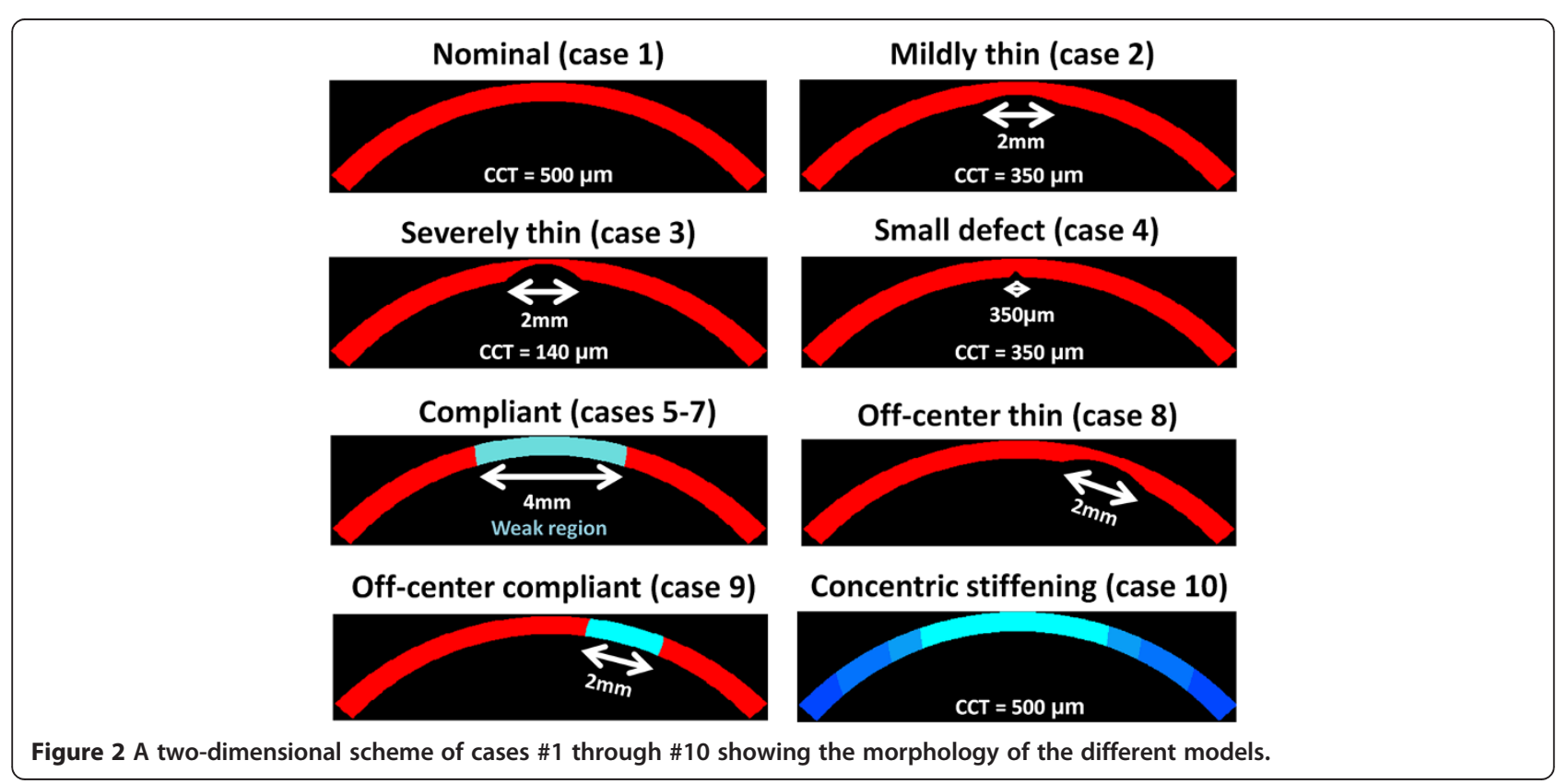




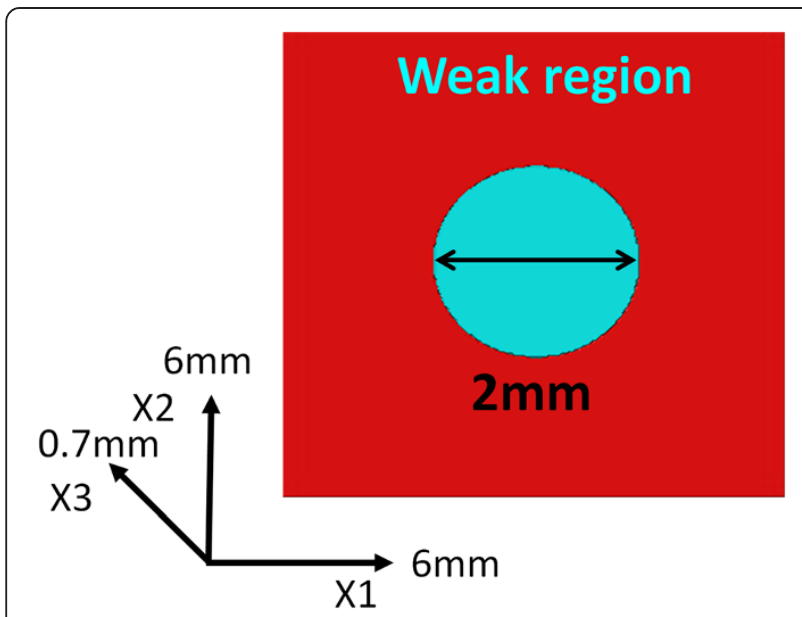

Figure 3 The model of a thin square plate with a thickness of $0.7 \mathrm{~mm}$ and sides of $6 \mathrm{~mm}$ was created with a $2 \mathrm{~mm}$ diametered weak region.

elements on the posterior surface were subjected to an IOP of $20 \mathrm{mmHg}$. The IOP levels used herein are considered to be within the normal physiological range $[1,9,12]$. The higher IOP value in the last three cases is used solely in order to emphasize the results for the thin plate configuration.

\section{Numerical method}

Mesh density was set based on the implementation of the local best fit sphere (BFS) algorithm on the nodes of the anterior surface of the cornea post-deformation (as explained later on). All cases were meshed semiautomatically, eventually including $100 \mathrm{~K}$ 4-node tetrahedron elements. In terms of numerical convergence or accuracy, using greater mesh densities did not provide any benefit (resulted in less than 1\% difference in strain data for denser meshes in preliminary analysis). The FE simulations were all set up and pre-processed using PreView (Ver. 1.8), analyzed using the Pardiso linear solver of FEBio (http://mrl.sci.utah.edu/software/febio) (Ver.

Table 2 A summarization of the mechanical properties for the bulk and weak region of the transversely isotropic and orthotropic cases

\begin{tabular}{lccccc}
\hline & \multicolumn{2}{c}{ Transversely isotropic } & & \multicolumn{2}{c}{ Orthotropic } \\
\cline { 2 - 3 } & Bulk & Weak region & & Bulk & Weak region \\
\hline E1 $[\mathrm{MPa}]$ & 2 & 1 & & 2 & 1 \\
E2 $[\mathrm{MPa}]$ & 2 & 1 & & 3.5 & 3.5 \\
E3 $[\mathrm{MPa}]$ & 4.5 & 4.5 & & 4.5 & 4.5 \\
G31 $[\mathrm{MPa}]$ & 0.2 & 0.2 & & 0.2 & 0.2 \\
G32 $[\mathrm{MPa}]$ & 0.2 & 0.1 & & 0.2 & 0.1 \\
V12 & 0.49 & 0.49 & & 0.49 & 0.49 \\
\hline
\end{tabular}

1.5.1) in structural mechanics mode, and post-processed using PostView (Ver. 1.4) [13]. The runtime of each case was approximately 5-10 min using a 64-bit Windows 7based workstation with a CPU comprising Intel Xeon E5645 2.4 GHz (2 processors), and 32Gb of RAM.

\section{Dioptric power maps}

Dioptric power maps are commonly used for identifying and describing corneal pathologies. In order to use them with our analysis, a dedicated computer code was written in MATLAB (The MathWorks, MATLAB 2012a) based on the BFS algorithm. The Cartesian coordinates of the anterior surface nodes post-deformation were imported. Then local groups of the neighboring nodes with a spherical radius of $3 \mathrm{~mm}$ for all the individual nodes on the anterior surface were created. Each group was fitted to a sphere using the criteria of minimal sum of squares. This results in a map of the best fit local radius of curvatures for each of the nodes on the anterior surface [1]. In order to validate the algorithm several spheres with known radiuses varying from $3 \mathrm{~mm}$ to $9 \mathrm{~mm}$ were tested with the defined mesh density, resulting in an accuracy of tenths of microns.

The dioptric power for each node is computed with the simplified Lensmakers' equation to produce the dioptric power maps:

$$
\operatorname{Diopter}\left(x_{i}, y_{i}, z_{i}\right)=\frac{337.5}{R_{i}} \forall i \in\{\text { Anterior surface }\}
$$

Where $x_{i}, y_{i}$ and $z_{i}$ are the Cartesian coordinates of the anterior surface nodes of the cornea post-deformation and $R_{i}$ is the radius of curvature that was calculated for each node.

\section{Outcome measures}

The post-deformation geometry of the anterior surfaces was extracted and the BFS algorithm was utilized to produce corresponding diopter maps for all simulations.

For cases \#1, \#3, \#7 and \#10, representing the nominal geometry, extreme degradation in thickness and mechanical properties and concentric stiffening, the center corneal elevation (CCE) was measured. For cases \#11 13 the maximum elevation was measured.

In cases \#1-3 and \#5-7 the diopter values in the center of the region were sampled in order to graph the dioptric change that is induced by modifying the corneal thickness and modulus of elasticity.

\section{Results}

The deformations and dioptric power maps for the nominal case (case \#1), along with both extreme cases of thinning and weakening (cases \#3 and \#7 respectively), 


\section{Case \#7, 90\% compliant}

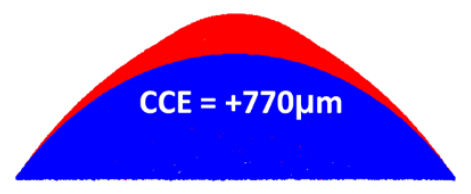

Case \#1, nominal

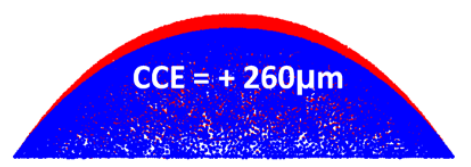

Case \#3, severely thin

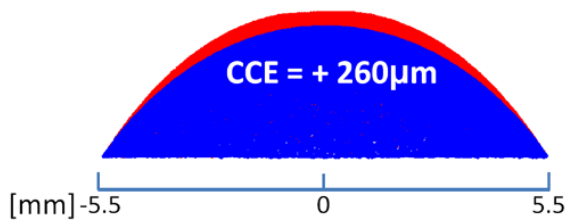

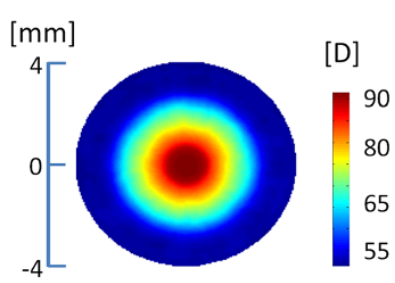
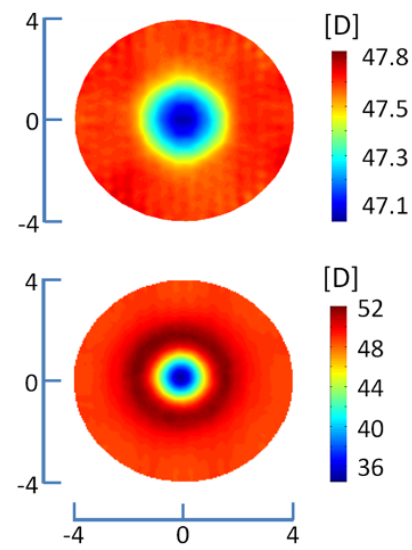

Figure $4 \mathrm{~A}$ side view of the deformations on an $11 \mathrm{~mm}$ domain alongside with the dioptric power maps on an 8 mm domain for cases \#1, \#3 and \#7. The center corneal elevation (CCE) is also presented for these cases. While the 90\% more compliant case (Case \#7) develops a bulge, the severely thin case (Case \#3) flattens. The nominal case shows some central flattening due to the natural thinning of the cornea towards the center region. Interestingly, the nominal case and case \#3 have identical elevations.

are shown in Figure 4. The difference in morphology between case \#7 and case \#3 (and therefore also the differences in the dioptric power maps) is easily noticeable. While the $90 \%$ more compliant case (Case \#7) develops a bulge at the center of the cornea, the severely thin case (Case \#3) flattens. The nominal case shows some central flattening due to the natural thinning of the cornea towards the center region. Interestingly, the nominal case and case \#3 have identical CCEs. While the deformations are shown for the entire corneal domain, the dioptric power maps are presented on an $8 \mathrm{~mm}$ domain as commonly displayed by standard topographers in order to emphasize the maximal optical region.

A CCE of 290 microns and a dioptric power map that is similar in morphology to the nominal case with diopters ranging between 46.8 and 48.6 was observed in case \#4 (small defect).

Decreasing the modulus of elasticity and thinning had an opposite effect on the dioptric power. For a cornea with a constant modulus of elasticity value of $0.4 \mathrm{MPa}$, an increase in thickness from 150 to 500 microns resulted in approximately 25 diopters increase. Maintaining the thickness at 500 microns and decreasing the stiffness from 0.4 $\mathrm{MPa}$ to $0.04 \mathrm{MPa}$ resulted in an increase of more than 40 diopters. Results for cases \#1-3 and cases \#5-7, are plotted in Figure 5. The left axis shows the minimal thickness of the cornea (marked with asterisks) and the right axis shows the modulus of elasticity (marked with hollow circles). The nominal case (case \#1) is where both plots coincide (i.e. a minimal thickness of 500 microns and a modulus of elasticity of 0.4 $\mathrm{MPa}$ ). Notice that the far left asterisk and the far right hollow circle represent cases \#3 and \#7 respectively.

In general, for homogenous isotropic properties (cases \#1 - 9), a thin region is characterized by low local diopter values in oppose to a weak region that is characterized by high ones. Cases \#8 and \#9 (off-center

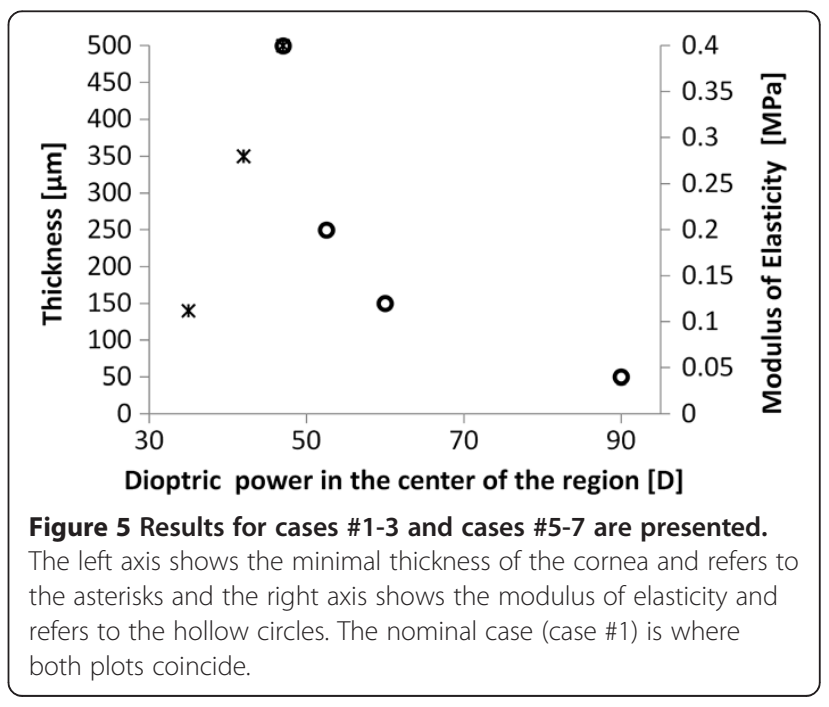


cases) exhibited the same trend as cases \#2 and \#7 respectively.

Comparing between the dioptric power maps of the nominal case and case \#10, it is clear that they have opposite trends regardless of the fact that both show similar maximal elevations. Namely, for the nominal case the center region has low diopter values that increase towards the limbus whereas, in case $\# 10$ the opposite is true (Figure 6).

Examining the results for the thin plate configuration, the maximum elevation in the isotropic case is larger than the transversely isotropic case which is also larger than the orthotropic case. Most interesting, the dioptric power maps reveal major differences; the central region (where the weak region is located) in the isotropic case exhibits an increase in diopters relative to the periphery whereas cases \#12 and \#13 show a decrease (Figure 7).

\section{Discussion}

Several studies have previously shown that subjecting the cornea to an increase in IOP results in an elevation of the anterior surface. Whether using computerized FEM or in-vitro using harvested corneas, this is occasionally measured as maximal tissue displacement or as a rise in apical displacement [1,14-17]. The CCE (cases \#1 - 10) and maximum elevation (cases \#11 - 13) increased accordingly. The CCE of the nominal case (case \#1), and both extreme cases of thinning and weakening (cases \#3 and \#7 respectively) shows the tendency of a weak region to develop larger elevations than a thin region (Figure 4). Comparing the CCE between the nominal case and the severely thin case emphasizes the importance of the dioptric power maps. These reveal major differences in the underlying mechanisms; while both cases \#1 and \#3 inflate under $10 \mathrm{mmHg}$ up to a CCE of $260 \mu \mathrm{m}$, the thin case flattens to an extent of 36 [D] whereas the minimal value for the nominal case was 47.1[D]. The importance of the dioptric power maps is further emphasized when examining the differences between cases \#1 and \#7. It is evident that while the maximum diopter value for the nominal case is located in the periphery with a gradual decrease towards the center of the cornea, the maximum diopter for the compliant case is in the center of the cornea and decreases towards the periphery. Observing the side view of the deformations, it is evident that there exists a tremendous difference between cases \#3 and \#7; the first flattens while the latter is bulges.

A numeric presentation of this phenomenon is presented in Figure 5, plotting among the above mentioned also cases \#2, \#5 and \#6. For a constant modulus of elasticity an increase in thickness results in an increase in diopter and for constant thickness a decrease in stiffness also results in an increase in diopter.

Cases \#4 (small defect), \#8 and \#9 (off-center thinning and mechanical degradation respectively) provide further generalizations; the small defect depicts that even when the thinning occurs in a tiny region the trend remains as in cases \#2 and \#3 and the off-center cases show that the difference in dioptric power maps between thin and weak regions is not constrained to corneal thinning and weakening in the central region only.

When the cornea's mechanical properties where defined as non-homogenous (i.e. concentric stiffening towards the limbus) (case \#10), the resulting CCE was lower than the nominal case by $50 \mu \mathrm{m}$, in accordance with an overall increase in stiffness. Interestingly, the highest diopter value was now located in the center of the cornea and decayed towards the limbus in oppose to the nominal case (Figure 6). A FEM analysis of a nonhomogenous orthotropic linear elastic cornea was previously presented in a biomechanical analysis of the keratoconic cornea [1]. The non-homogeneity was manifested by a gradual increase in the circumferential elastic moduli towards the limbus. When a local thin region was simulated it resulted in a rise in the dioptric power as well as a rise in the maximal tissue displacement as obtained herein in case \#10. The gradual increase in the circumferential elastic moduli towards the limbus seems to have enormous implications on the shape of the cornea and therefore on its dioptric power. Namely, for a given thickness under a constant IOP, a sudden loss of the non-homogeneity characteristic would result in flattening of the center of the cornea due to the fact that

\section{Case \#10, Concentric stiffening}

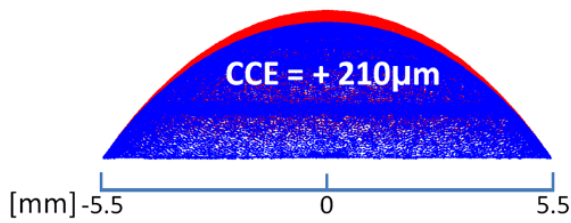

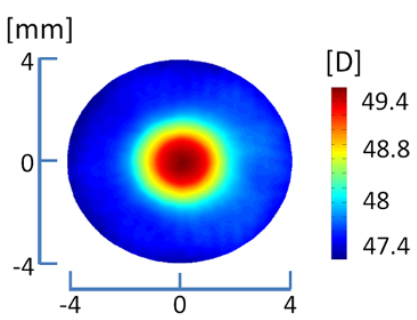

Figure 6 A side view of the deformations on an $11 \mathrm{~mm}$ domain and the dioptric power maps for cases \#10. The center corneal elevation is also noted. 


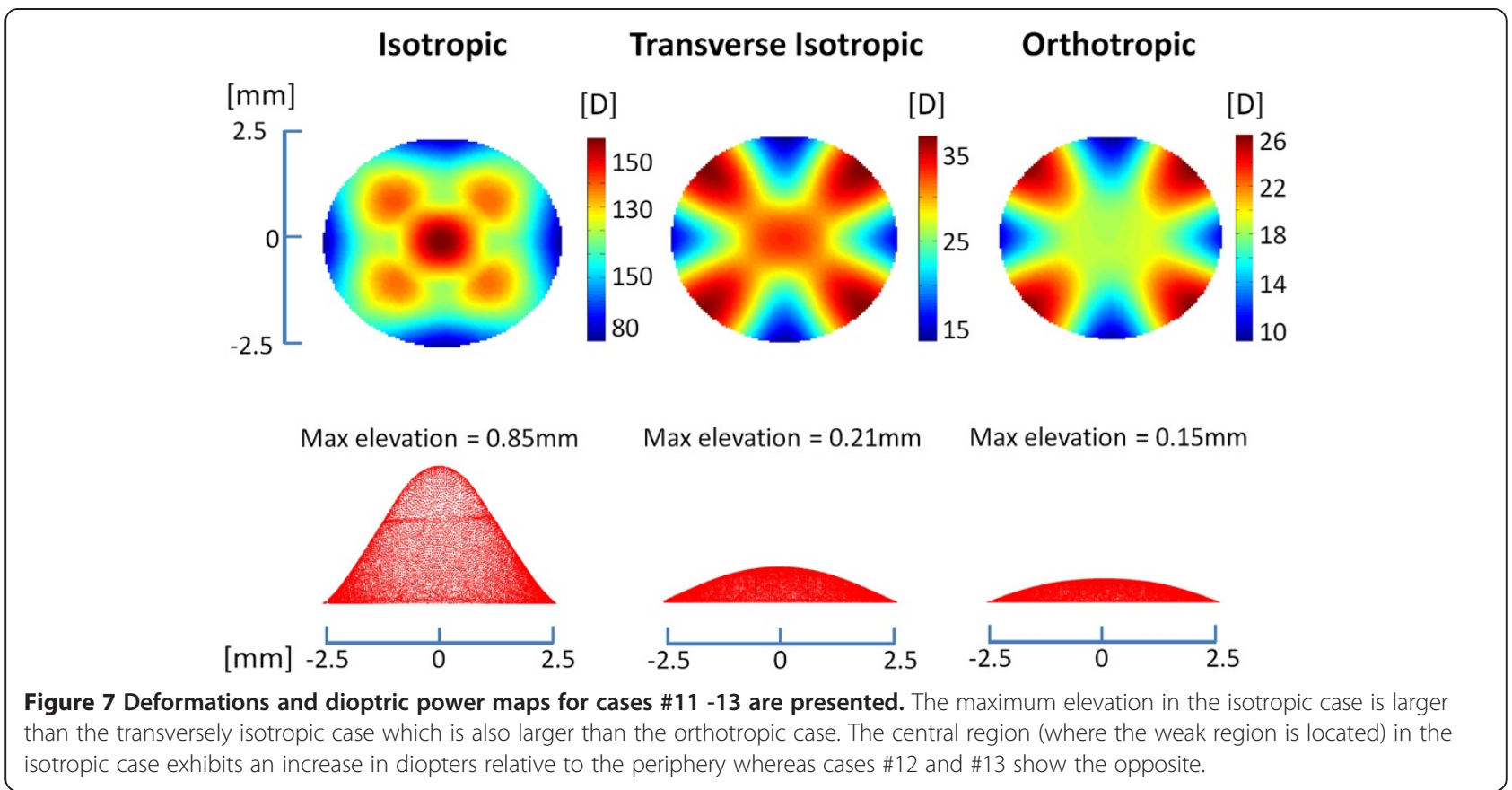

the cornea is naturally thinnest at its center (a transition from case \#10 to case \#1). Mind that such transition would be characterized by an increase in meridional stress in the center of the cornea. Previous studies which present more advanced constitutive modeling of the cornea such as orthotropic linear elasticity [1] and hyperelastic anisotropy [14] simulated keratoconus by defining weak regions which resulted in similar surface elevations and dioptric power maps to those obtained in case \#10. Simulation of a thin region resulted in a decrease in diopter as depicted by case \#2 [1].

The plate simulations (cases \#11 - 13) show an increase in maximal elevations from orthotropic to isotropic through transversely isotropic mechanical properties according to expectations. Nevertheless, these basic simulations reveal an important difference between the dioptric maps; while the maximal dioptric value for the isotropic case is in the center of the plate it is not as such for cases \#12 and \#13. These cases show increased flattening towards the center of the plate with a larger difference for the latter. It is a known fact that the orthotropic characteristics of the cornea are derived from the underlying collagen fiber orientation $[1,3,18]$. A sudden loss of directionality of the fibers that are oriented, for instance, in the circumferential direction would result in a transfer from case \#13 to case \#12 and if the meridional directioned fibers are also suddenly dispersed, a further transition from case \#12 to case \#11 would occur.

Considering that sudden changes in both the nonhomogeneous and orthotropic characteristics of the cornea are unlikely, the following quasi static process is proposed describing a cascade in which the cornea develops a bulged region: Begin with a normal cornea that has healthy geometry and mechanical properties. If the circumferential fibers weaken in such a way that encourages homogeneity, the center of the cornea, where it is thinnest, would develop elevated meridional stresses due to the tendency of that region to flatten (illustrated and supported by the transition from case \#10 to case \#1). Though only minor deformations might be visible at this stage, the stress would weaken the meridional fibers that are located in the center region resulting in a slight corneal elevation due to a transition from transversal isotropic to isotropic (illustrated and supported by the transition from case \#12 to \#11). The slight elevation is accompanied by minor thinning which induces more stress on the meridional fibers, now due to the increasing tendency to flatten (illustrated and supported by case \#2). The central region becomes a little more isotropic and slightly elevates more. The graduate transition to isotropic mechanical properties induces gradual thinning and bulging and vice versa. This positive feedback loop could cease when a full isotropic central region is reached (illustrated and supported by Case \#5).

According to the proposed pathogenesis, the changes in geometry are secondary to changes in mechanical properties. The cornea's mechanical properties have been measured in-vitro resulting in great variability primarily due to the non-homogeneous orthotropic visco-hyperelastic behavior it exerts but also owing to the differences in the experimental setups (strips/whole corneas, uni/ bi-axial stretching or inflation with several clamping mechanisms), time post-mortem, storage conditions 
and the biological variability (age, pathology etc.). A good assessment of corneal biomechanics in-vivo is even more difficult to obtain. One interesting measure termed as the corneal resistance factor (CRF) was introduced with the Reichert's Ocular response analyzer (Reichert Ophthalmic Instruments, Buffalo, NY) and captures the cornea's resistance to deformation $[19,20]$. Nevertheless, the CRF has not been compared with traditional mechanical variables, including the various elastic moduli or viscoelastic coefficients $[19,20]$. Though it might be possible in the future to assess the corneal biomechanical coefficients in-vivo, the difficulty to do so in the present weakens the possibility of identifying the early onset of keratoconus with these data. Based on the proposed process, it could be possible to capture the early stages of keratoconus by analyzing the corneal fiber distribution alone. The loss of circumferential fiber orientation is identified to be a primary biomechanical factor in the process of creating the bulge. Certain degrees of dispersion of the meridional fiber orientation in the center of the cornea could be an indicator for the progressed stage of the pathology. Evidence to this possibility that is based on x-ray scattering techniques in-vitro was previously published [18]. Abnormalities in the distribution of aligned collagen were observed in the peripheral region of keratoconic corneas. The extent of the disruption was reported to be independent of the disease severity and the size and shape of the cone. The author also mentions that the absence of the skewed diamond shape arrangement of aligned collagen in keratoconus suggests that the anchoring fibrils are not present [18].

The adaptation of existing optical characteristics (such as the corneal birefringence [21]) to identify fiber orientation and dispersion in the cornea in-vivo may prove to be most beneficial for identifying the early stages of keratoconus. This would allow quick interventional crosslinking strategies that can delay the deterioration of the mechanical properties and therefore the progress of the pathology.

The present models are based on several assumptions which impose inherent limitations that should be considered when interpreting the results. First, all models were based on elastic constitutive laws whereas the cornea is known to exhibit hyperelastic mechanical properties. Nevertheless, hyperelasticity is more relevant for IOPs that are above the ones simulated herein. Second, the non-homogenous case (case \#10) is represented by concentric stiffening of an isotropic material whereas the majority of reinforcing fibers in the cornea are in the circumferential direction. Nonetheless, the results that were obtained for case \#10 are supported by previous studies that have considered the concentric stiffening solely in the circumferential direction $[1,14]$. Finally, the orthotropic plate is a preliminary configuration that differs greatly from the morphology of the cornea. Still, the results show a convincing trend that can very well have an impact also on the geometry of the cornea.

\section{Conclusions}

To conclude, a biomechanical process in which the cornea gradually develops a conic like shape, similar to that appearing in keratoconus was presented. A healthy cornea that is subjected to normal IOP will bulge in reaction to degradation of the non-homogenous circumferential fiber distribution. This is because when these fibers weaken the center of the cornea develops increasing meridional stresses that could in turn weaken the in plane fibers in that region. This would result in a small elevation of the center of the cornea followed by center corneal thinning which would further degrade and weaken the reinforcing fibers. Early intervention based on an in-vivo assessment of corneal fiber distributions might become possible.

\section{Competing interests}

The authors declare that they have no competing interests.

\section{Authors' contributions}

RA developed the modeling and performed the simulations. All authors, RA, $A G, D V$ and EM, participated in the analysis and interpretation of data, and all authors drafted the manuscript. All authors read and approved the final manuscript.

\section{Author details}

'Department of Biomedical Engineering, Faculty of Engineering, Tel Aviv University, Tel Aviv 69978, Israel. ²Department of Ophthalmology, Tel Aviv Medical Center, Sackler Faculty of Medicine, Tel-Aviv University, Tel-Aviv 69978, Israel.

Received: 6 September 2013 Accepted: 17 December 2013 Published: 23 October 2014

\section{References}

1. Gefen A, Shalom R, Elad D, Mandel Y (2009) Biomechanical analysis of the keratoconic cornea. J Mech Behav Biomed Mater 2(3):224-236

2. Pinsky PM, Datye DV (1991) A microstructurally-based finite element model of the incised human cornea. J Biomech 24(10):907-922

3. Grytz R, Meschke G (2010) A computational remodeling approach to predict the physiological architecture of the collagen fibril network in corneo-scleral shells. Biomech Model Mechanobiol 9(2):225-235

4. Boote C, Dennis S, Meek K (2004) Spatial mapping of collagen fibril organisation in primate cornea-an X-ray diffraction investigation. J Struct Biol 146(3):359-367

5. Boote C, Dennis S, Huang Y, Quantock AJ, Meek KM (2005) Lamellar orientation in human cornea in relation to mechanical properties. J Struct Biol 149(1):1-6

6. Li LY, Tighe B (2006) The anisotropic material constitutive models for the human cornea. J Struct Biol 153(3):223-230

7. Andreassen T, Simonsen AH, Oxlund H (1980) Biomechanical properties of keratoconus and normal corneas. Exp Eye Res 31(4):435-441

8. Edmund C (1989) Corneal topography and elasticity in normal and keratoconic eyes. A methodological study concerning the pathogenesis of keratoconus. Acta Ophthalmol Suppl 193:1-36

9. Asher R, Gefen A, Varssano D (2012) Patient-Specific Modeling of the Cornea. In: Gefen A (ed), Patient-Specific Modeling in Tomorrow's Medicine, vol 9. Springer Berlin Heidelberg, Germany, pp 461-483. ISBN 978-3-642-24618-0

10. Rabinowitz YS (1998) Keratoconus. Surv Ophthalmol 42:297-319

11. Varssano D, Asher R, Gefen A (2012) Biomechanical Modeling of the Human Eye with a Focus on the Cornea. In: E.Y.K. Ng, CRC Press (eds), MultiModality State-of-the-Art: Human Eye Imaging and Modeling, Boca Raton, FL, USA. ISBN 9781439869932

12. Pandolfi A, Fotia G, Manganiello F (2008) Finite element simulations of laser refractive corneal surgery. Eng Comput 25(1):15-24 
13. Maas SA, Ellis BJ, Ateshian GA, Weiss JA (2012) FEBio: finite elements for biomechanics. J Biomech Eng 134(1):011005

14. Pandolfi A, Manganiello $F$ (2006) A model for the human cornea: constitutive formulation and numerical analysis. Biomech Model Mechanobiol 5(4):237-246

15. Elsheikh A, Geraghty B, Rama P, Campanelli M, Meek KM (2010) Characterization of age-related variation in corneal biomechanical properties. J R Soc Interface 7:1475-1485

16. Elsheikh A, Wang D, Brown M, Rama P, Campanelli M, Pye D (2007) Assessment of corneal biomechanical properties and their variation with age. Curr Eye Res 32:11-19

17. Liu J, Roberts CJ (2005) Influence of corneal biomechanical properties on intraocular pressure measurement: quantitative analysis. J Cataract Refract Surg 31:146-155

18. Hayes S, Boote C, Tuft SJ, Quantock AJ, Meek KM (2007) A study of corneal thickness, shape and collagen organisation in keratoconus using videokeratography and X-ray scattering techniques. Exp Eye Res 84:423-434

19. Lau W, Pye D (2011) A clinical description of ocular response analyzer measurements. Invest Ophthalmol Vis Sci 52:2911-2916

20. Luce DA (2005) Determining in vivo biomechanical properties of the cornea with an ocular response analyzer. J Cataract Refract Surg 31:156-162

21. Götzinger E, Pircher M, Sticker M, Dejaco-Ruhswurm I, Kaminski S, Skorpik C, Fercher AF, Hitzenberger CK (2003) Pathologic changes of corneal birefringence imaged with polarization sensitive optical coherence tomography. Invest Ophthalmol Vis Sci 44:3668, E-Abstract 3668

doi:10.1186/2196-050X-1-3

Cite this article as: Asher et al:: Etiology of Keratoconus: proposed biomechanical pathogenesis. In Silico Cell and Tissue Science 2014 1:3.

\section{Submit your manuscript to a SpringerOpen ${ }^{\circ}$ journal and benefit from:}

- Convenient online submission

- Rigorous peer review

- Immediate publication on acceptance

- Open access: articles freely available online

- High visibility within the field

- Retaining the copyright to your article

Submit your next manuscript at $\gg$ springeropen.com 